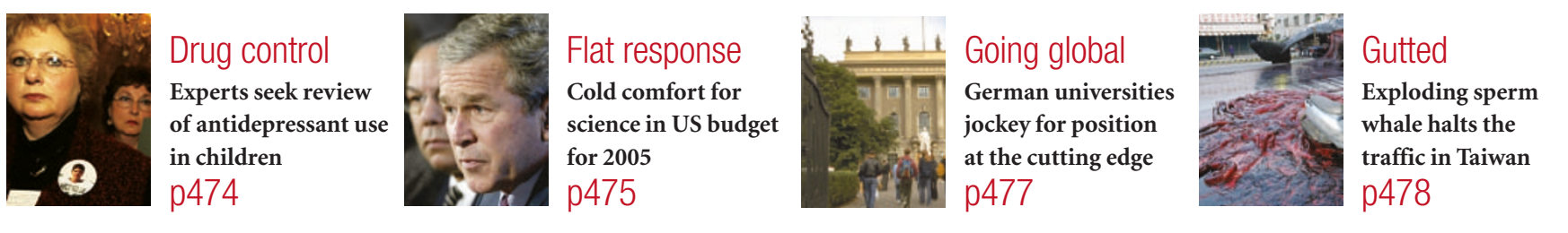

\title{
Fear of human pandemic grows as bird flu sweeps through Asia
}

\section{Alison Abbott and Helen Pearson}

Public-health officials and lab researchers are this week engaged in a frantic battle to contain the escalating outbreak of bird flu in Asia, and to minimize the risk of it developing into a human flu pandemic.

The first cases of apparent human-to-human transmission of the avian flu virus were reported in Vietnam on 1 February, heightening public-health concerns. As officials struggle to contain the avian outbreak by orchestrating the massacre of chicken flocks, researchers around the world are preparing the way for the mass production of a vaccine that could help to contain a human pandemic.

Individual human-to-human transmission of the virus doesn't necessarily mean that it has evolved into a strain that will transmit readily enough to cause a significant outbreak, never mind a pandemic. But should a pandemic occur, many experts say that current public-health plans are unlikely to prevent large-scale loss of life. Previous human flu pandemics, such as the 1918 outbreak that killed about 40 million people, originated in birds.

The present outbreak of the highly pathogenic $\mathrm{H} 5 \mathrm{~N} 1$ virus - named for the

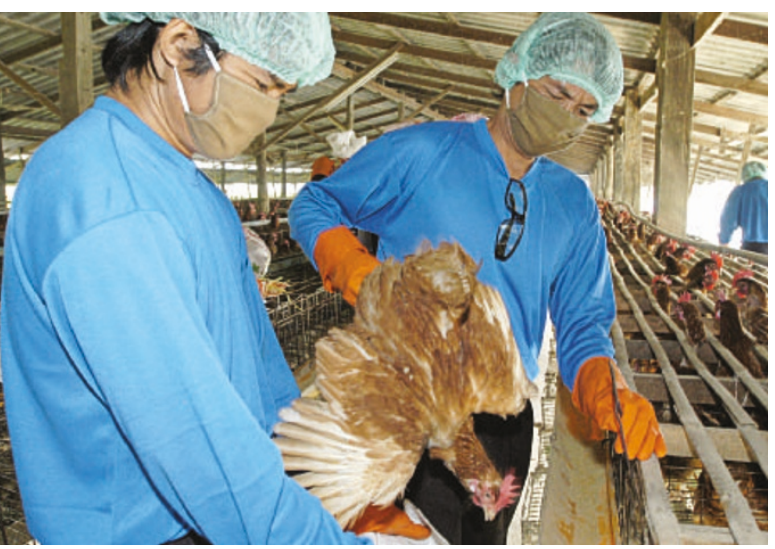

Cutting the links: health officials are culling chickens in a concerted effort to halt the spread of bird flu to humans.

easily between people (see 'Mixed messages', opposite). Officials don't put much stock in vaccinating the region's vast chicken flocks, especially as this would not eliminate infectivity - healthy birds that are still shedding the virus would undermine the effort.

The World Health Organization (WHO) is working on the first stage of a five-point action plan for dealing with pandemic influenza, says Klaus Stöhr, project leader of the organization's global influenza programme. This involves seeking to contain the avian outbreak while monitoring human-to-human transmission.

The US Centers for Disease

subtypes of its key surface proteins, haemagglutinin and neuraminidase — has ripped through flocks of domestic poultry in southeast Asia and spread to at least 14 people, killing 11 of them.

In efforts to curb the spread of the virus, the eight countries affected so far (see map, opposite) have slaughtered 20 million chickens. Experts say that culling infected birds is the only way to extinguish the virus before it infects more people. This can reduce the risk that avian and normal human flu meet and exchange genes in an individual, creating a strain with the ability to spread
Control and Prevention (CDC) in Atlanta, Georgia, a laboratory at St Jude Children's Research Hospital in Memphis, Tennessee, and the National Institute for Biological Standards and Control in London are working with the WHO to prepare a human vaccine. They expect a prototype $\mathrm{H} 5 \mathrm{~N} 1$ vaccine strain to be engineered shortly and safetytested in ferrets within two months. This will then be handed to manufacturers for clinical testing, which should take up to another two months. If the virus starts spreading between people, mass production would begin, which would take an extra four to six months.

\section{Lack of infrastructure hampers virus monitoring}

In the capital of Cambodia, Phnom Penh, only six adults and children have been tested for influenza this winter. "Routine surveillance is just not possible," says Sean Tobin, a World Health Organization epidemiologist stationed there.

That's the reality facing public-health officials in the poorer countries where avian flu may or may not mutate into a human pandemic. And for all their talk of control and mitigation strategies, health officials acknowledge that there's little that can be done on the ground in Cambodia, Laos or other countries that lack even a rudimentary public healthcare system.

According to a spokesman for the World Organisation for Animal Health, Laos and Cambodia lack many of the essentials needed to control an outbreak, including sufficient numbers of veterinarians, transport to get samples to the laboratory - or indeed laboratories themselves. He says that infected populations of chickens will continue to go undetected in these countries.

Rapid diagnostic tests to identify avian flu in chickens are available at national centres in Vietnam and Thailand, but not in their poorer neighbours. In Cambodia, it took almost two weeks from the detection of suspected cases of avian virus in chickens - with subsequent tests done by the Pasteur Institute in Paris - for the government to confirm the report. It took officials in Laos eight days after suspected cases were detected before they could announce results from laboratories in Thailand and Vietnam.

Cambodia and Laos are also unable to test for human influenza subtypes. These tests are needed to isolate patients infected with avian virus from those with the normal flu.

Last year's SARS epidemic was a learning experience for Cambodia, says Tobin. "Some health officials in the provinces are used to working with case definitions that we provide," he says. "But getting information down to the provincial level is notoriously difficult in this country." And whereas the main SARS risk was restricted to areas visited by foreigners, the risk of avian flu is nationwide, affecting regions where public health resources are at their most meagre. 


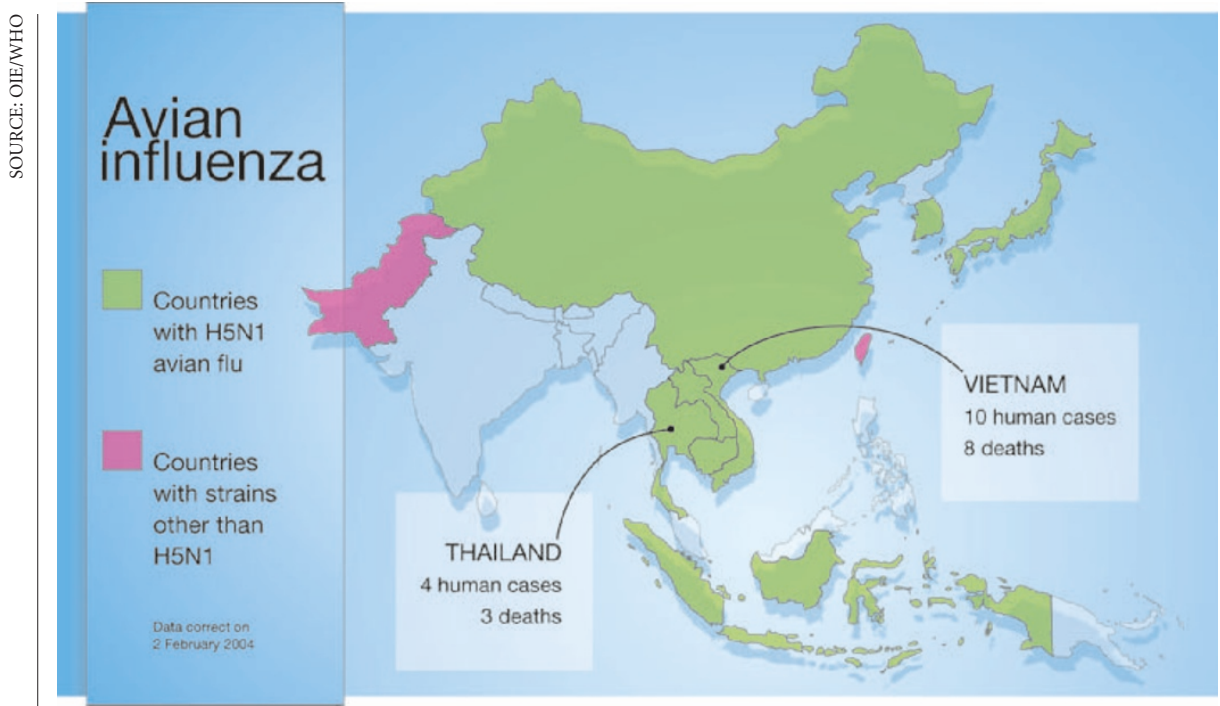

The lengthy production schedule means that in the worst-case scenario, the first wave of a pandemic could almost be over before a vaccine is ready, some experts warn. "Most of the damage will have been done by the time a vaccine is widely available," says Rino Rappuoli, head of research at Chiron Vaccines in Siena, Italy, one of the major companies in Europe working on pandemic flu vaccines. Even so, a vaccine might be useful in fighting any subsequent 'waves' of the pandemic.

Stöhr concedes that "vaccines will not be ready for those at the epicentre of any pandemic". Antiviral drugs will be the first line of medical intervention, he says, but these may also be in short supply. Preliminary tests done by the CDC have shown that the H5N1 virus is resistant to a traditional group of drugs, including amantadine and rimantadine, that block virus replication.

\section{Short supply}

A newer and more expensive class of drug that cripples the neuraminidase enzyme in flu viruses is effective against H5N1 strains. The most common version of these drugs is oseltamivir (Tamiflu), made by Swiss-based drug company Roche. But the company won't say how many doses of Tamiflu it has or reveal its capacity to manufacture more, says spokesman Terence Hurley. He claims that Roche is cooperating with the WHO and offering Tamiflu at a reduced price for emergency use in poor countries.

Other experts doubt whether stocks of Tamiflu would be sufficient in the face of a pandemic. "It's in very limited supply and would quickly run out," says epidemiologist Ira Longini at Emory University in Atlanta, Georgia, who has modelled Tamiflu's potential impact. Prompted by the severe flu season this winter, the CDC is already building up a significant stockpile of Tamiflu. Japan and some European countries are also negotiating with Roche to get stocks of the drug.

Meanwhile, efforts in southeast Asia are aimed at lowering the rate of transmission to humans by culling chickens as quickly and safely as possible. Stöhr is concerned that many of those doing the culling don't have the recommended protective clothing, and aren't being vaccinated against the circulating strain of normal human flu, or taking prophylactic antiviral drugs. These steps would reduce the chances of the avian flu combining with human flu strains in their bodies. "We need to get rid of the natural reservoir of $\mathrm{H} 5 \mathrm{~N} 1$, but we need to do it safely," says Stöhr. outbreak will add impetus to their calls for governments to be better prepared. They would like to see improved surveillance of circulating strains of animal flu, large-scale manufacture of vaccines to possible pandemic strains before human-to-human transmission is seen, or stockpiling of antiviral drugs.

Many countries are drafting such schemes. The German health authorities, for example, are finalizing a plan that would routinely prepare prototype vaccine strains based on subtypes of the haemagglutinin surface proteins circulating in animals or birds. "This could save months in vaccine preparation if a pandemic threat arises in the future," says Reinhard Kurth, president of the Robert Koch Institute in Berlin.

The Canadian government has already implemented part of its pandemic influenza plan: ithas a contract with Quebec-based Shire Biologics that requires the company to be ready to manufacture a pandemic vaccine. The company could produce 6 million doses per month once it is supplied with the prototype vaccine strain, says Health Canada spokesperson Emmanuel Chabot in Ottawa, Ontario.

Other countries should invest in similar schemes to ready themselves for future flu pandemics, experts say. "We are going to face this over and over again," predicts publichealth researcher Scott Layne of the University of California, Los Angeles.

For more coverage see:

www.nature.com/nature/focus/birdflu
Some researchers hope that the current

\section{Mixed messages}

Public-health officials badly need to know whether a strain of flu virus is likely to emerge that can spread readily from people to people - but researchers can't tell them. This knowledge gap is stirring debate about whether animals should be co-infected with bird and human flu viruses in the laboratory in an attempt to get a better grip on the biological mechanisms involved.

"It would give us some idea of how concerned we should be," says Richard Webby, a virologist at St Jude Children's Research Hospital in Memphis, Tennessee. "But it's a question of whether these experiments should be done - you would potentially be creating a pandemic virus."

A strain of avian flu that could be transmitted to humans might emerge if the avian H5N1 virus and an existing human flu strain both infect the same cell in the same person or bird. Mixing of genes from the two strains could then result in a human-transmissible virus. "It's a random event that we can't just predict, given what we currently know," says David Heymann, an infectious-disease expert at the World Health Organization in Geneva.

Efforts to model the scenario on a computer are hampered by a lack of information on how many birds and people harbour each virus. Additionally, says virologist Robert Webster, also at St Jude, "We know zippo about what genes are involved in transmissibility."

Researchers are worried that it is only a matter of time before the two viruses meet and mix. And some think that the number of people infected with avian flu in southeast Asia is greatly underestimated by official statistics.

"The more exposure to avian viruses, the more chance you would have that such an event would occur," says virologist Albert Osterhaus of Erasmus University in Rotterdam, the Netherlands.

Human and avian influenza viruses are already being mixed in labs that research flu vaccines - but only using non-pathogenic strains of human virus and less virulent forms of the bird virus. To model the pandemic threat, researchers need to know the conditions under which viruses mix their genes to generate the most deadly combinations.

"It is possible to create a virus with pandemic potential," says Osterhaus. But such an experiment "would have to be done in an extremely high-security laboratory", says Heymann. "You could create a monster."

And some are sceptical of what would be gained from better laboratory data. Real pandemics are influenced by stochastic events that can't be predicted, points out Roy Anderson, an epidemiologist at Imperial College, London, who is trying to model the probability of such combinations. "It's exceedingly difficult and I'm not optimistic that we'll produce something of value," he says. Carina Dennis 